\title{
Supporting Information \\ A-B-A-B-A Block Amphiphiles. Balance between Hydrophilic and Hydrophobic Segmentation
}

\author{
Fredric M. Menger*, Hao Lu, and Dan Lundberg \\ Department of Chemistry, Emory University, Atlanta, GA 30322 \\ menger@emory.edu
}

\begin{abstract}
Materials
All reagents were purchased from Aldrich or Bachem and used without additional purification. All solvents used were reagent or HPLC grade and dried over 4 Å molecular sieves.

Characterization Methods

${ }^{1} \mathrm{H}$ and ${ }^{13} \mathrm{C}$ NMR spectra were acquired on a Varian INOVA $400 \mathrm{MHz}\left(100 \mathrm{MHz}\right.$ for $\left.{ }^{13} \mathrm{C}\right)$ instrument. Mass spectra experiments were completed by the Emory University Mass Spectrometry Center. Surface tension measurements were conducted on a Fisher Surface Tensiomat following the Du Nouy ring procedure. Elemental analyses were performed by Atlantic Microlab in Norcross, GA.
\end{abstract}

\section{Syntheses of $E_{m} C_{n} E_{m} C_{n} E_{m}$.

$$
\mathrm{H}\left(\mathrm{OCH}_{2} \mathrm{CH}_{2}\right)_{3} \mathrm{OH}+\mathrm{TrCl} \stackrel{\text { Pyridine }}{\longrightarrow} \mathrm{H}\left(\mathrm{OCH}_{2} \mathrm{CH}_{2}\right)_{3} \mathrm{OTr}
$$

Under $\mathrm{N}_{2}, 10.109 \mathrm{~g} \mathrm{TrCl}$ (MW 278.78, 36.3mmol) was added into a 100ml 3-neck round bottom flask charged with $62.6 \mathrm{ml} \mathrm{H}\left(\mathrm{OCH}_{2} \mathrm{CH}_{2}\right)_{3} \mathrm{OH}(70.429 \mathrm{~g}, 363 \mathrm{mmol})$ and $4.302 \mathrm{~g}$ pyridine $(54.4 \mathrm{mmol})$ and the reaction was stirred at $45^{\circ} \mathrm{C}$ overnight. The reaction mixture was poured into a separatory funnel and $70 \mathrm{ml}$ distilled water was added. The mixture was shaken vigorously and allowed to settle for $3 \mathrm{~h}$. The bottom layer was separated from the aqueous solution, and the aqueous solution was extracted with toluene. The bottom layer was dissolved in toluene and combined with the toluene extract. The toluene solution was washed with distilled water and dried over $\mathrm{MgSO}_{4}$ for $1 \mathrm{~h}$ with stirring, filtered, and concentrated to give gel-like crude. The crude was purified by flash chromatography (hexanes/ EtOAc 2:1) and gave 13.578g (95\%) colorless gel-like product: ${ }^{1} \mathrm{H} \mathrm{NMR}\left(\mathrm{CDCl}_{3}, 400 \mathrm{MHz}\right) \delta 2.29(\mathrm{~b}, 1 \mathrm{H}), 3.26(\mathrm{t}, J=5.2,2 \mathrm{H}), 3.63-3.74$ $(\mathrm{m}, 10 \mathrm{H}), 7.22-7.32(\mathrm{~m}, 9 \mathrm{H}), 7.47-7.48(\mathrm{~d}, J=7.6,6 \mathrm{H}) ;{ }^{13} \mathrm{C} \mathrm{NMR}\left(\mathrm{CDCl}_{3}, 400 \mathrm{MHz}\right) \delta 62.03,63.47,70.76,70.90$, $71.02,72.68,86.77,127.14,127.96,128.89,144.26 .^{1}$

$$
\mathrm{TrOE}_{3} \mathrm{OH}+\mathrm{HOOC}\left(\mathrm{CH}_{2}\right)_{10} \mathrm{COOH} \stackrel{\mathrm{DCC}, \mathrm{DMAP}}{\longrightarrow} \mathrm{TrOE}_{3} \mathrm{OOC}\left(\mathrm{CH}_{2}\right)_{10} \mathrm{COOH}
$$

Under $\mathrm{N}_{2}$, one $250 \mathrm{ml}$ round bottom flask was charged with $2.00 \mathrm{~g} \mathrm{TrOE}_{3} \mathrm{OH}$ (MW 392.49, 5.1mmol), 1.15g 1,10decanedicarboxlic acid (MW 230.30, 5.0mmol), 60mg DMAP (MW 122.17, 0.5mmol), 120ml dry $\mathrm{CH}_{2} \mathrm{Cl}_{2}$, and a stirrer bar. Via a pressure-equalized addition funnel was added dropwise $1.03 \mathrm{~g}$ DCC (MW 206.33, 5.0mmol) in 70ml dry $\mathrm{CH}_{2} \mathrm{Cl}_{2}$ over $1 \mathrm{hr}$. The reaction was stirred for another 5 hours at r.t. The reaction was filtered and concentrated to give crude. The crude was dissolved in $30 \mathrm{ml}$ acetone, filtered again, and concentrated to give oil crude. The oil crude was subjected to flash chromatography (EtOAC/ $\left.\mathrm{CH}_{2} \mathrm{Cl}_{2} 8: 1\right)$ to give colorless gel-like product $(1.39 \mathrm{~g}$, $48 \%)$ : ${ }^{1} \mathrm{H} \mathrm{NMR}$ $\left(\mathrm{CDCl}_{3}, 400 \mathrm{MHz}\right) \delta 1.27(\mathrm{~b}, 12 \mathrm{H}), 1.64-1.63(\mathrm{~m}, 4 \mathrm{H}), 2.84-2.37(\mathrm{~m}, 4 \mathrm{H}), 3.28(\mathrm{t}, J=5.2,2 \mathrm{H}), 3.69-3.75(\mathrm{~m}, 8 \mathrm{H}), 4.24$ $(\mathrm{t}, J=4.8,2 \mathrm{H}), 7.22-7.32(\mathrm{~m}, 9 \mathrm{H}), 7.47-7.49(\mathrm{~d}, J=7.6,6 \mathrm{H}) ;{ }^{13} \mathrm{C} \mathrm{NMR}\left(\mathrm{CDCl}_{3}, 400 \mathrm{MHz}\right) \delta 24.85,25.03,29.21,29.25$, $29.37,29.51,34.20,34.35,63.49,63.60,69.47,70.84,70.90,70.96,86.73,127.11,127.95,128.89,144.27,174.12$, 179.80 .

\section{$\operatorname{TrOE}_{3} \mathrm{OOC}\left(\mathrm{CH}_{2}\right){ }_{10} \mathrm{COOH}+\mathrm{HOE}_{3} \mathrm{OH} \stackrel{\text { DCC, DMAP }}{\longrightarrow} \operatorname{TrOE}_{3} \mathrm{OOC}\left(\mathrm{CH}_{2}\right)_{10} \mathrm{COOE}_{3} \mathrm{OOC}\left(\mathrm{CH}_{2}\right)_{10} \mathrm{COOE}_{3} \mathrm{OTr}$}

Under $\mathrm{N}_{2}$, a 50ml round bottom flask was charged with 966mg monoester (MW 604.34, 1.6mmol), 155mg tri(ethylene glycol) (MW 194.23, 0.8mmol), 330mg DCC (MW 206.33, 1.6mmol), 20mg DMAP (MW 122.17, 0.16mmol), and stirring bar in $30 \mathrm{ml} \mathrm{CH}_{2} \mathrm{Cl}_{2}$. The reaction was carried overnight at r.t., and filtered, concentrated to give crude. The crude was dissolved in $20 \mathrm{ml}$ acetone, and filtered again, concentrated to give gel-like crude. Flash chromatography on silica $\left(\mathrm{CH}_{2} \mathrm{Cl}_{2} /\right.$ EtOAc 8:1 $\rightarrow$ 2:1) gave colorless gel-like product $(510 \mathrm{mg}, 48 \%):{ }^{1} \mathrm{H} \mathrm{NMR}\left(\mathrm{CDCl}_{3}, 400 \mathrm{MHz}\right) \delta 1.24(\mathrm{~b}$, 
$24 \mathrm{H}), 1.58-1.60(\mathrm{~m}, 8 \mathrm{H}), 2.25-2.33(\mathrm{~m}, 8 \mathrm{H}), 3.23(\mathrm{t}, J=5.2,4 \mathrm{H}), 3.64-3.72(\mathrm{~m}, 24 \mathrm{H}), 4.20-4.22(\mathrm{~m}, 8 \mathrm{H}), 7.19-7.30(\mathrm{~m}$, $18 \mathrm{H}), 7.43-7.46(\mathrm{~m}, 12 \mathrm{H}) ;{ }^{13} \mathrm{C} \mathrm{NMR}\left(\mathrm{CDCl}_{3}, 400 \mathrm{MHz}\right) \delta 25.09,29.32,29.45,29.59,34.38,34.36,63.51,63.60,69.45$, 69.49, 70.74, 70.89, 70.91, 70.99, 86.72, 127.12, 127.96, 128.90, 144.29, 174.02.

$$
\operatorname{TrOE}{ }_{3} \mathrm{OOC}\left(\mathrm{CH}_{2}\right)_{10} \mathrm{COOE}_{3} \mathrm{OOC}\left(\mathrm{CH}_{2}\right)_{10} \mathrm{COOE}_{3} \mathrm{OTr} \stackrel{\mathrm{H}_{2}, \mathrm{Pd} / \mathrm{C}}{\longrightarrow} \mathrm{HOE}_{3} \mathrm{OOC}\left(\mathrm{CH}_{2}\right)_{10} \mathrm{COOE}_{3} \mathrm{OOC}\left(\mathrm{CH}_{2}\right)_{10} \mathrm{COOE}_{3} \mathrm{OH}
$$

$\mathbf{E}_{3} \mathbf{C}_{10} \mathbf{E}_{\mathbf{3}} \mathbf{C}_{10} \mathbf{E}_{3}$ : One $25 \mathrm{ml}$ round bottom flask was charged with $240 \mathrm{mg}$ ditrityl-protected ester (MW 1322.75, $0.18 \mathrm{mmol}$ ), $5 \mathrm{mg} 5 \% \mathrm{Pd} / \mathrm{C}, 15 \mathrm{ml} \mathrm{CH}_{2} \mathrm{Cl}_{2}$, and a stirrer bar. Hydrogenolysis was carried out at room temperature under $1 \sim 2 \mathrm{~atm}$ of $\mathrm{H}_{2}$ for 24 hours using a balloon. Upon completion of the reaction, the catalyst was filtered and washed with $\mathrm{CH}_{2} \mathrm{Cl}_{2}$. The filtrate was concentrated and subjected to flash chromatography on silica (Hexanes/EtOAc 1:1 $\rightarrow$ Hexanes/EtOAc /MeOH 1:1:0.2). The product was collected as white solid (141mg, 92\%): ${ }^{1} \mathrm{H} \mathrm{NMR}\left(\mathrm{CDCl}_{3}, 400 \mathrm{MHz}\right)$ $\delta 1.28(\mathrm{~b}, 24 \mathrm{H}), 1.58-1.63(\mathrm{~m}, 8 \mathrm{H}), 2.14(\mathrm{~b}, 2 \mathrm{H}), 2.31-2.35(\mathrm{~m}, 8 \mathrm{H}), 3.61-3.75(\mathrm{~m}, 28 \mathrm{H}), 4.22-4.25(\mathrm{~m}, 8 \mathrm{H}) ;{ }^{13} \mathrm{C} \mathrm{NMR}$ $\left(\mathrm{CDCl}_{3}, 400 \mathrm{MHz}\right) \delta 25.09,29.32,29.45,29.59,34.39,61.97,63.41,63.51,69.42,69.45,70.55,70.75,72.681,174.08$; HRMS Calcd. for $\mathrm{C}_{42} \mathrm{H}_{78} \mathrm{NaO}_{16}[\mathrm{M}+\mathrm{Na}]^{+}$861.5188, found 861.5166. Anal. Calcd.(\%) for $\mathrm{C}_{42} \mathrm{H}_{78} \mathrm{O}_{16}+1 / 2 \mathrm{H}_{2} \mathrm{O}: \mathrm{C}$, 59.48; H, 9.39; O, 31.13. Found: C, 59.71; H, 9.35; O, 31.01.

\section{$\mathrm{TrOE}_{3} \mathrm{OH}+\mathrm{HOOC}\left(\mathrm{CH}_{2}\right)_{8} \mathrm{COOH} \stackrel{\text { DCC, DMAP }}{\longrightarrow} \operatorname{TrOE}_{3} \mathrm{OOC}\left(\mathrm{CH}_{2}\right)_{8} \mathrm{COOH}$}

Under $\mathrm{N}_{2}$, one $250 \mathrm{ml}$ round bottom flask was charged with 2.62g $\mathrm{TrOE}_{3} \mathrm{OH}$ (MW 392.49, $6.7 \mathrm{mmol}$ ), 1.35g sebacic acid (MW 202.25, 6.68mmol), 82mg DMAP (MW 122.17, 0.67mmol), 130ml dry $\mathrm{CH}_{2} \mathrm{Cl}_{2}$, and a stirrer bar. Via a pressure-equalized addition funnel was added dropwise 1.38g DCC (MW 206.33, 6.7mmol) in 70ml dry $\mathrm{CH}_{2} \mathrm{Cl}_{2}$ over 1 hr. The reaction was stirred for another 5 hours at r.t. The reaction was filtered and concentrated to give crude. The crude was dissolved in $30 \mathrm{ml}$ acetone, filtered again, and concentrated to give oil crude. The oil crude was subjected to flash chromatography (EtOAC/ $\left.\mathrm{CH}_{2} \mathrm{Cl}_{2} 3: 1\right)$ to give colorless gel-like product $(1.83 \mathrm{~g}, 48 \%)$ : ${ }^{1} \mathrm{H} \mathrm{NMR}\left(\mathrm{CDCl}_{3}\right.$, $400 \mathrm{MHz}) \delta 1.27(\mathrm{~b}, 8 \mathrm{H}), 1.57-1.62(\mathrm{~m}, 4 \mathrm{H}), 2.27-2.34(\mathrm{~m}, 4 \mathrm{H}), 3.24(\mathrm{t}, J=5.6,2 \mathrm{H}), 3.67-3.73(\mathrm{~m}, 8 \mathrm{H}), 4.22(\mathrm{t}, J=5.2$, $2 \mathrm{H}), 7.19-7.30(\mathrm{~m}, 9 \mathrm{H}), 7.45-7.47(\mathrm{~d}, J=8.0,6 \mathrm{H}) ;{ }^{13} \mathrm{C} \mathrm{NMR}\left(\mathrm{CDCl}_{3}, 400 \mathrm{MHz}\right) \delta 24.70,24.88,29.02,29.09,34.11$, $34.20,63.38,63.52,69.33,70.71,70.80,70.84,86.63,127.01,127.84,128.78,144.17,174.02,179.99$.

\section{$\mathrm{TrOE}_{3} \mathrm{OOC}\left(\mathrm{CH}_{2}\right)_{8} \mathrm{COOH}+\mathrm{HOE}_{3} \mathrm{OH} \stackrel{\text { DCC, DMAP }}{\longrightarrow} \operatorname{TrOE}_{3} \mathrm{OOC}\left(\mathrm{CH}_{2}\right)_{8} \mathrm{COOE}_{3} \mathrm{OOC}\left(\mathrm{CH}_{2}\right)_{8} \mathrm{COOE}_{3} \mathrm{OTr}$}

Under $\mathrm{N}_{2}$, a $100 \mathrm{ml}$ round bottom flask was charged with $3.23 \mathrm{~g}$ monoester (MW 576.72, 5.6mmol), 0.54g tri(ethylene glycol) (MW 194.23, 2.8mmol), 1.16g DCC (MW 206.33, 5.6mmol), 35mg DMAP (MW 122.17, 0.28mmol), 380mg HOBt (MW 135.13, 2.8mmol), and stirring bar in $50 \mathrm{ml} \mathrm{CH}_{2} \mathrm{Cl}_{2}$. The reaction was kept at $0{ }^{\circ} \mathrm{C}$ for two hours and overnight at r.t., and filtered, concentrated to give crude. The crude was dissolved in $20 \mathrm{ml}$ acetone, and filtered again, concentrated to give gel-like crude. Flash chromatography on silica $\left(\mathrm{CH}_{2} \mathrm{Cl}_{2} / \mathrm{EtOAc}\right.$ 5:1) gave colorless gel-like product $(1.54 \mathrm{~g}, 43 \%):{ }^{1} \mathrm{H}$ NMR $\left(\mathrm{CDCl}_{3}, 400 \mathrm{MHz}\right) \delta 1.26(\mathrm{~b}, 16 \mathrm{H}), 1.58-1.60(\mathrm{~m}, 8 \mathrm{H}), 2.25-2.33(\mathrm{~m}, 8 \mathrm{H}), 3.23(\mathrm{t}, J=$ $5.2,4 \mathrm{H}), 3.64-3.72(\mathrm{~m}, 24 \mathrm{H}), 4.20(\mathrm{t}, J=4.8,8 \mathrm{H}), 7.19-7.30(\mathrm{~m}, 18 \mathrm{H}), 7.44-7.47(\mathrm{~m}, 12 \mathrm{H}) ;{ }^{13} \mathrm{C} \mathrm{NMR}\left(\mathrm{CDCl}_{3}\right.$, $400 \mathrm{MHz}) \delta 24.95,24.97,29.20,34.24,34.27,63.44,63.54,69.37,69.41,70.66,70.80,70.84,70.92,86.65,127.05$, $127.89,128.83,144.23,173.92$.

$$
\operatorname{TrOE}{ }_{3} \mathrm{OOC}\left(\mathrm{CH}_{2}\right)_{8} \mathrm{COC}\left(\mathrm{CH}_{2}\right)_{8} \mathrm{COOE}_{3} \mathrm{OTr} \stackrel{\mathrm{H}_{2}, \mathrm{Pd} / \mathrm{C}}{\longrightarrow} \mathrm{HOE}_{3} \mathrm{OOC}\left(\mathrm{CH}_{2}\right)_{8} \mathrm{COOE}_{3} \mathrm{OOC}\left(\mathrm{CH}_{2}\right)_{8} \mathrm{COOE}_{3} \mathrm{OH}
$$

$\mathbf{E}_{3} \mathbf{C}_{8} \mathbf{E}_{3} \mathbf{C}_{8} \mathbf{E}_{3}$ : One $50 \mathrm{ml}$ round bottom flask was charged with $1.47 \mathrm{~g}$ ditrityl-protected ester (MW 1267.58, 1.16mmol), $28 \mathrm{mg} 5 \% \mathrm{Pd} / \mathrm{C}, 30 \mathrm{ml} \mathrm{CH}_{2} \mathrm{Cl}_{2}$, and a stirrer bar. Hydrogenolysis was carried out at room temperature under 1 2 atm of $\mathrm{H}_{2}$ for 36 hours using a balloon. Upon completion of the reaction, the catalyst was filtered and washed with $\mathrm{CH}_{2} \mathrm{Cl}_{2}$. The filtrate was concentrated and subjected to flash chromatography on silica (Hexanes/EtOAc 1:1 $\rightarrow$ Hexanes/EtOAc $/ \mathrm{MeOH} 1: 1: 0.2)$. The product was collected as colorless oil $(860 \mathrm{mg}, 95 \%):{ }^{1} \mathrm{H} \mathrm{NMR}\left(\mathrm{CDCl}_{3}, 400 \mathrm{MHz}\right) \delta 1.23(\mathrm{~b}, 16 \mathrm{H})$, $1.528-1.56(\mathrm{~m}, 8 \mathrm{H}), 2.23-2.28(\mathrm{~m}, 8 \mathrm{H}), 2.70(\mathrm{~b}, 2 \mathrm{H}), 3.53-3.66(\mathrm{~m}, 28 \mathrm{H}), 4.14-4.17(\mathrm{~m}, 8 \mathrm{H}) ;{ }^{13} \mathrm{C} \mathrm{NMR}\left(\mathrm{CDCl}_{3}\right.$, $400 \mathrm{MHz}) \delta 24.84,29.04,29.07,34.13,61.69,63.25,63.34,69.18,69.23,70.34,70.55,72.54,173.80$; HRMS Calcd. for $\mathrm{C}_{38} \mathrm{H}_{70} \mathrm{NaO}_{16}[\mathrm{M}+\mathrm{Na}]^{+}$805.4562, found 805.4542. Anal. Calcd.(\%) for $\mathrm{C}_{38} \mathrm{H}_{70} \mathrm{O}_{16}$ : C, 58.29; H, 9.01; O, 32.70 . Found: C, 58.13; H, 9.08; O, 32.42.

$$
\mathrm{TrOE}_{3} \mathrm{OH}+\mathrm{HOOC}\left(\mathrm{CH}_{2}\right)_{6} \mathrm{COOH} \stackrel{\mathrm{DCC}, \mathrm{DMAP}}{\longrightarrow} \operatorname{TrOE}_{3} \mathrm{OOC}\left(\mathrm{CH}_{2}\right)_{6} \mathrm{COOH}
$$

Under $\mathrm{N}_{2}$, one $250 \mathrm{ml}$ round bottom flask was charged with 7.67g $\mathrm{TrOE}_{3} \mathrm{OH}$ (MW 392.49, $19.6 \mathrm{mmol}$ ), 3.41g suberic acid (MW 174.2, 19.6mmol), 240mg DMAP (MW 122.17, 1.96mmol), 120ml dry $\mathrm{CH}_{2} \mathrm{Cl}_{2}$, and a stirrer bar. Via a pressure-equalized addition funnel was added dropwise 4.04g DCC (MW 206.33, 19.6mmol) in 60ml dry $\mathrm{CH}_{2} \mathrm{Cl}_{2} \mathrm{over}$ $1 \mathrm{hr}$. The reaction was stirred for another 6 hours at r.t. The reaction was filtered and concentrated to give crude. The crude was dissolved in $30 \mathrm{ml}$ acetone, filtered again, and concentrated to give oil crude. The oil crude was subjected to flash chromatography (EtOAC/ $\left.\mathrm{CH}_{2} \mathrm{Cl}_{2} 3: 1\right)$ to give colorless gel-like product $(1.83 \mathrm{~g}, 42 \%)$ : ${ }^{1} \mathrm{H} \mathrm{NMR}\left(\mathrm{CDCl}_{3}\right.$, $400 \mathrm{MHz}) \delta 1.32-1.33(\mathrm{~m}, 4 \mathrm{H}), 1.60-1.64(\mathrm{~m}, 4 \mathrm{H}), 2.29-2.34(\mathrm{~m}, 4 \mathrm{H}), 3.26(\mathrm{t}, J=3.2,2 \mathrm{H}), 3.69-3.75(\mathrm{~m}, 8 \mathrm{H}), 4.24(\mathrm{t}, J$ 
$=3.2,2 \mathrm{H}), 7.22-7.31(\mathrm{~m}, 9 \mathrm{H}), 7.47-7.49(\mathrm{~d}, J=5.2,6 \mathrm{H}) ;{ }^{13} \mathrm{C} \mathrm{NMR}\left(\mathrm{CDCl}_{3}, 400 \mathrm{MHz}\right) \delta 24.62,24.78,28.80,34.02$, $34.18,63.47,63.64,69.46,70.83,70.89,70.96,86.72,127.11,127.94,128.89,144.28,173.93,179.53$.

\section{$\operatorname{TrOE}{ }_{3} \mathrm{OOC}\left(\mathrm{CH}_{2}\right)_{6} \mathrm{COOH}+\mathrm{HOE}_{3} \mathrm{OH} \stackrel{\mathrm{DCC}, \mathrm{DMAP}}{\longrightarrow} \operatorname{TrOE}_{3} \mathrm{OOC}\left(\mathrm{CH}_{2}\right)_{6} \mathrm{COOE}_{3} \mathrm{OOC}\left(\mathrm{CH}_{2}\right)_{6} \mathrm{COOE}_{3} \mathrm{OTr}$}

Under $\mathrm{N}_{2}$, a $100 \mathrm{ml}$ round bottom flask was charged with 3.50g monoester (MW 548.67, 6.37mmol), 0.62g tri(ethylene glycol) (MW 194.23, 3.2mmol), 1.31g DCC (MW 206.33, 6.37mmol), 40mg DMAP (MW 122.17, 0.32mmol), 220mg $\mathrm{HOBt}$ (MW 135.13, 1.6mmol), and stirring bar in $50 \mathrm{ml} \mathrm{CH}_{2} \mathrm{Cl}_{2}$. The reaction was kept at $0{ }^{\circ} \mathrm{C}$ for two hours and overnight at r.t., and filtered, concentrated to give crude. The crude was dissolved in $20 \mathrm{ml}$ acetone, and filtered again, concentrated to give gel-like crude. Flash chromatography on silica $\left(\mathrm{CH}_{2} \mathrm{Cl}_{2} / \mathrm{EtOAc} 4: 1\right)$ gave colorless gel-like product $(1.74 \mathrm{~g}, 45 \%):{ }^{1} \mathrm{H} \mathrm{NMR}\left(\mathrm{CDCl}_{3}, 400 \mathrm{MHz}\right) \delta 1.25(\mathrm{~b}, 8 \mathrm{H}), 1.57-1.60(\mathrm{~m}, 8 \mathrm{H}), 2.26-2.31(\mathrm{~m}, 8 \mathrm{H}), 3.23(\mathrm{t}, J=$ $2.8,4 \mathrm{H}), 3.63-3.71(\mathrm{~m}, 24 \mathrm{H}), 4.21(\mathrm{t}, J=2.8,8 \mathrm{H}), 7.19-7.28(\mathrm{~m}, 18 \mathrm{H}), 7.45-7.46(\mathrm{~m}, 12 \mathrm{H}) ;{ }^{13} \mathrm{C} \mathrm{NMR}\left(\mathrm{CDCl}_{3}\right.$, $400 \mathrm{MHz}) \delta 24.79,28.84,34.18,63.45,63.56,69.35,69.39,70.66,70.81,70.83,70.92,86.65,127.05,127.89,128.83$, $144.23,173.77$.

$$
\operatorname{TrOE}_{3} \mathrm{OOC}\left(\mathrm{CH}_{2}\right)_{6} \mathrm{COOE}_{3} \mathrm{OOC}\left(\mathrm{CH}_{2}\right)_{6} \mathrm{COOE}_{3} \mathrm{OTr} \stackrel{\mathrm{H}_{2}, \mathrm{Pd} / \mathrm{C}}{\longrightarrow} \mathrm{HOE}_{3} \mathrm{OOC}\left(\mathrm{CH}_{2}\right)_{6} \mathrm{COOE}_{3} \mathrm{OOC}\left(\mathrm{CH}_{2}\right)_{6} \mathrm{COOE}_{3} \mathrm{OH}
$$

$\mathbf{E}_{3} \mathbf{C}_{6} \mathbf{E}_{3} \mathbf{C}_{6} \mathbf{E}_{3}$ : One $50 \mathrm{ml}$ round bottom flask was charged with $1.62 \mathrm{~g}$ ditrityl-protected ester (MW 1211.46, 1.34mmol), $32 \mathrm{mg} 5 \% \mathrm{Pd} / \mathrm{C}, 30 \mathrm{ml} \mathrm{CH}_{2} \mathrm{Cl}_{2}$, and a stirrer bar. Hydrogenolysis was carried out at room temperature under 1 2 atm of $\mathrm{H}_{2}$ for 36 hours using a balloon. Upon completion of the reaction, the catalyst was filtered and washed with $\mathrm{CH}_{2} \mathrm{Cl}_{2}$. The filtrate was concentrated and subjected to flash chromatography on silica (Hexanes/EtOAc 1:1 $\rightarrow$ Hexanes/EtOAc $/ \mathrm{MeOH} 1: 1: 0.2)$. The product was collected as colorless oil $(885 \mathrm{mg}, 91 \%):{ }^{1} \mathrm{H}$ NMR $\left(\mathrm{CDCl}_{3}, 400 \mathrm{MHz}\right) \delta 1.31-1.34(\mathrm{~m}$, $8 \mathrm{H}), 1.60-1.63(\mathrm{~m}, 8 \mathrm{H}), 2.30-2.34(\mathrm{~m}, 8 \mathrm{H}), 3.59-3.73(\mathrm{~m}, 28 \mathrm{H}), 4.20-4.24(\mathrm{~m}, 8 \mathrm{H}) ;{ }^{13} \mathrm{C} \mathrm{NMR}\left(\mathrm{CDCl}_{3}, 400 \mathrm{MHz}\right)$ $\delta$ 24.83, 28.90, 34.21, 61.91, 63.41, 63.52, 69.35, 69.39, 70.49, 70.71, 72.68, 173.89; HRMS Calcd. for $\mathrm{C}_{34} \mathrm{H}_{62} \mathrm{NaO}_{16}$ $[\mathrm{M}+\mathrm{Na}]^{+}$749.3936, found 749.3933. Anal. Calcd.(\%) for $\mathrm{C}_{34} \mathrm{H}_{62} \mathrm{O}_{16}+1 / 2 \mathrm{H}_{2} \mathrm{O}: \mathrm{C}, 55.50 ; \mathrm{H}, 8.63 ; \mathrm{O}, 35.88$. Found: C, 55.54; H, 8.76; O, 35.69 .

$$
\mathrm{H}\left(\mathrm{OCH}_{2} \mathrm{CH}_{2}\right)_{6} \mathrm{OH}+\mathrm{TrCl} \stackrel{\text { Pyridine }}{\longrightarrow} \mathrm{H}\left(\mathrm{OCH}_{2} \mathrm{CH}_{2}\right)_{6} \mathrm{OTr}
$$

Under $\mathrm{N}_{2}, 8.2 \mathrm{TrCl}$ (MW 278.78, 29.4mmol) was added into a 100ml 3-neck round bottom flask charged with $25 \mathrm{~g}$ $\mathrm{H}\left(\mathrm{OCH}_{2} \mathrm{CH}_{2}\right)_{3} \mathrm{OH}(\mathrm{MW} 282.34,88 \mathrm{mmol})$ and $10 \mathrm{ml}$ pyridine and the reaction was stirred at $45^{\circ} \mathrm{C}$ overnight. After the reaction is completed, $200 \mathrm{ml}$ DI water was added and $3 \mathrm{x} 100 \mathrm{ml}$ toluene was used to extract the product. The combined organic solution was dried over $\mathrm{Na}_{2} \mathrm{SO}_{4}$ and the solvent was removed to give crude. Flash chromatography $(5 \% \mathrm{MeOH}$ in EtOAc) resulted 13.6g colorless product $(88 \%):{ }^{1} \mathrm{H} \mathrm{NMR}\left(\mathrm{CDCl}_{3}, 400 \mathrm{MHz}\right) \delta 3.24(\mathrm{t}, J=4.4,2 \mathrm{H}), 3.59-3.73(\mathrm{~m}$, $22 \mathrm{H}), 7.21-7.31(\mathrm{~m}, 9 \mathrm{H}), 7.46-7.48(\mathrm{~d}, J=7.6,6 \mathrm{H}) ;{ }^{13} \mathrm{C} \mathrm{NMR}\left(\mathrm{CDCl}_{3}, 400 \mathrm{MHz}\right) \delta 61.94,63.53,70.53,70.77,70.82$, $70.89,70.99,71.50,72.74,86.73,127.13,127.98,128.93,144.34$.

\section{$\operatorname{TrOE}_{6} \mathrm{OH}+\mathrm{HOOC}\left(\mathrm{CH}_{2}\right){ }_{10} \mathrm{COOH} \stackrel{\text { EDCl, DMAP }}{\longrightarrow} \operatorname{TrOE}_{6} \mathrm{OOC}\left(\mathrm{CH}_{2}\right)_{10} \mathrm{COOH}$}

Under $\mathrm{N}_{2}$, one $250 \mathrm{ml}$ round bottom flask was charged with $5.72 \mathrm{~g} \mathrm{TrOE} 6 \mathrm{OH}$ (MW 524.65, 10.9mmol), 5.02g 1,10decanedicarboxlic acid (MW 230.30, 21.8mmol), 266mg DMAP (MW 122.17, 2.18mmol), 150ml dry THF, and a stirrer bar. After all the 1,10-decanedicarboxlic acid dissolved, 4.179g EDCI (MW 191.71, 21.8mmol) was added. The reaction was stirred for two days and the solution was filtered. The filtrate then was concentrated to give crude. Flash chromatography (7:1 DCM/ EtOAc) resulted $2.57 \mathrm{~g}$ colorless product $(32 \%):{ }^{1} \mathrm{H} \mathrm{NMR}\left(\mathrm{CDCl}_{3}, 400 \mathrm{MHz}\right) \delta 1.26(\mathrm{~b}$, $12 \mathrm{H}), 1.59-1.60(\mathrm{~m}, 4 \mathrm{H}), 2.29-2.33(\mathrm{~m}, 4 \mathrm{H}), 3.22(\mathrm{t}, J=4.8,2 \mathrm{H}), 3.62-3.68(\mathrm{~m}, 20 \mathrm{H}), 4.20(\mathrm{t}, J=4.8,2 \mathrm{H}), 7.19-7.29$ (m, 9H), 7.44-7.46 (d, $J=7.2,6 \mathrm{H}) ;{ }^{13} \mathrm{C} \mathrm{NMR}\left(\mathrm{CDCl}_{3}, 400 \mathrm{MHz}\right) \delta 24.70,24.86,29.03,29.06,29.19,29.33,34.01$, $34.16,63.29,63.36,69.14,70.49,70.53,70.55,70.65,70.74,86.51,126.92,127.75,128.69,144.10,173.87,178.86$.

$\operatorname{TrOE}_{6} \mathrm{OOC}\left(\mathrm{CH}_{2}\right)_{10} \mathrm{COOH}+\mathrm{HOE}_{6} \mathrm{OH} \stackrel{\text { EDCl, DMAP }}{\longrightarrow} \operatorname{TrOE}_{6} \mathrm{OOC}\left(\mathrm{CH}_{2}\right)_{10} \mathrm{COOE}_{6} \mathrm{OOC}\left(\mathrm{CH}_{2}\right)_{10} \mathrm{COOE}_{6} \mathrm{OTr}$

Under $\mathrm{N}_{2}$, a $50 \mathrm{ml}$ round bottom flask was charged with $715 \mathrm{mg}$ monoester (MW 736.95, 0.97mmol), $136 \mathrm{mg}$ hexta(ethylene glycol) (MW 282.34, 0.48mmol), 372mg EDCI (MW 191.71, 1.94mmol), 24mg DMAP (MW 122.17, $0.19 \mathrm{mmol})$, and stirring bar in $30 \mathrm{ml}$ dry THF. The reaction was carried overnight at r.t., and filtered, concentrated to give crude. Preparative TLC (EMD, $20 \mathrm{~cm} \times 20 \mathrm{~cm} \times 2 \mathrm{~mm}, 3: 1 \mathrm{CH}_{2} \mathrm{Cl}_{2}$ : EtOAc) was used to separate crude mixture and resulted 231mg colorless gel-like product $(28 \%):{ }^{1} \mathrm{H} \mathrm{NMR}\left(\mathrm{CDCl}_{3}, 400 \mathrm{MHz}\right) \delta 1.25(\mathrm{~b}, 24 \mathrm{H}), 1.57-1.60(\mathrm{~m}, 8 \mathrm{H})$, 2.28-2.31 (m, 8H), $3.20(\mathrm{t}, J=5.6,4 \mathrm{H}), 3.61-3.68(\mathrm{~m}, 64 \mathrm{H}), 4.18-4.21(\mathrm{~m}, 8 \mathrm{H}), 7.18-7.28(\mathrm{~m}, 18 \mathrm{H}), 7.43-7.46(\mathrm{~m}$, $12 \mathrm{H}) ;{ }^{13} \mathrm{C} \mathrm{NMR}\left(\mathrm{CDCl}_{3}, 400 \mathrm{MHz}\right) \delta 25.03,29.27,29.40,29.55,34.33,63.45,63.50,69.33,70.72,70.81,70.93,86.65$, 127.06, 127.90, 128.85, 144.26, 173.98; HRMS Calcd. for $\mathrm{C}_{98} \mathrm{H}_{143} \mathrm{O}_{25}[\mathrm{M}+\mathrm{H}]^{+}$1719.9935, found 1719.9935.

$\mathrm{TrOE}_{6} \mathrm{OOC}\left(\mathrm{CH}_{2}\right){ }_{10} \mathrm{COOE}_{6} \mathrm{OOC}\left(\mathrm{CH}_{2}\right){ }_{10} \mathrm{COOE}_{6} \mathrm{OTr} \stackrel{\mathrm{H}_{2}, \mathrm{Pd} / \mathrm{C}}{\longrightarrow} \mathrm{HOE}_{6} \mathrm{OOC}\left(\mathrm{CH}_{2}\right)_{10} \mathrm{COOE} 6 \mathrm{OOC}\left(\mathrm{CH}_{2}\right)_{10} \mathrm{COOE}_{6} \mathrm{OH}$ 
$\mathbf{E}_{6} \mathbf{C}_{10} \mathbf{E}_{6} \mathbf{C}_{10} \mathbf{E}_{6}$ : One $25 \mathrm{ml}$ round bottom flask was charged with $350 \mathrm{mg}$ ditrityl-protected ester (MW 1720.16, 0.2mmol), $5 \mathrm{mg} 5 \% \mathrm{Pd} / \mathrm{C}, 15 \mathrm{ml} \mathrm{CH}_{2} \mathrm{Cl}_{2}$, and a stirrer bar. Hydrogenolysis was carried out at room temperature under 1 2 atm of $\mathrm{H}_{2}$ for 48 hours using a balloon. Upon completion of the reaction, the catalyst was filtered and washed with $\mathrm{CH}_{2} \mathrm{Cl}_{2}$. The filtrate was concentrated and subjected to flash chromatography on silica (Hexanes/EtOAc 1:1 $\rightarrow$ Hexanes/EtOAc $/ \mathrm{MeOH}$ 1:1:0.2). The product was collected as colorless oil $(226 \mathrm{mg}, 90 \%):{ }^{1} \mathrm{H} \mathrm{NMR}\left(\mathrm{CDCl}_{3}, 400 \mathrm{MHz}\right) \delta 1.81(\mathrm{~b}$, $24 \mathrm{H}), 1.50-1.53(\mathrm{~m}, 8 \mathrm{H}), 2.21-2.25(\mathrm{~m}, 8 \mathrm{H}), 3.52-3.66(\mathrm{~m}, 64 \mathrm{H}), 4.11-4.17(\mathrm{~m}, 8 \mathrm{H}) ;{ }^{13} \mathrm{C} \mathrm{NMR}\left(\mathrm{CDCl}_{3}, 400 \mathrm{MHz}\right)$ $\delta 24.89,29.09,29.24,29.38,34.16,34.19,61.12,63.25,63.35,69.17,69.34,69.84,70.11,70.16,70.23,70.31,70.55$, 70.58, 72.28, 173.82; HRMS Calcd. for $\mathrm{C}_{60} \mathrm{H}_{114} \mathrm{NaO}_{25}[\mathrm{M}+\mathrm{Na}]^{+}$1257.7547, found 1257.7504. Anal. Calcd. $(\%)$ for $\mathrm{C}_{60} \mathrm{H}_{114} \mathrm{O}_{25}$ : C, 58.33; H, 9.30; O, 32.37. Found: C, 55.45; H, 8.92; O, 32.48. Note: EA was taken on material recovered after all the experiments on it were completed.

\section{NMR Diffusion Experiments}

All NMR experiments were performed at $25^{\circ} \mathrm{C}$ on a Varian INOVA 600 spectrometer equipped with a pulsed field gradient (PFG) generator and a PFG amplifier. The samples were inserted into the probe at least 20 minutes prior to the experiments to allow for thermal equilibrium to be attained.

The experiments were run using a Hahn-echo sequence with intervening pulsed field gradients. ${ }^{2}$ The delay between the gradient pulses $(\Delta)$ and the width of the pulsed gradient pulses $(\delta)$ were kept constant at $140 \mathrm{~ms}$ and $7 \mathrm{~ms}$, respectively, while the strength of the pulsed gradient $(G)$ was linearly incremented from 0.01 up to $0.2 \mathrm{~T} / \mathrm{m}$ (maximum varied among experiments and samples) in 16 steps. The gradient strength was calibrated by making a measurement on $\mathrm{H}_{2} \mathrm{O}$ in $\mathrm{D}_{2} \mathrm{O}\left(D=1.902 * 10^{-9} \mathrm{~m}^{2} / \mathrm{s}\right)$, and linearity of the gradient amplifier in the used gradient strength interval was verified by measurements on poly(ethylene glycols) with known $D^{3}$.

The self-diffusion coefficients $(D)$ of solutes were calculated from the attenuation of the relevant echo peaks by a linear least-squares fit to the Stejskal-Tanner equation ${ }^{4}$ :

$\ln \left(I / I_{0}\right)=-(\gamma G \delta)^{2} D(\Delta-\delta / 3) \quad[1]$

where $I$ is the measured signal intensity, $I_{0}$ the signal intensity in the absence of gradient pulses, $\gamma$ the magnetogyric ratio of protons, and the rest of the parameters as defined above. In all experiments, the observed echo-decays gave very good fits to Eq. 1.

\section{References}

(1): Chen, Y.; Baker, G.L. J. Org. Chem.1999, 64, 6870.

(2): Price W.S. Concepts Magn. Res. 1997, 9, 299; 1998, 10, 197.

(3): Håkanson B.; Nydén M.; Söderman O. Colloid Polym. Sci. 2000, 278, 399.

(4): Stejskal E. O.; Tanner J. E. J. Chem. Phys. 1965, 42, 288. 